Tiefe, dass Weimar keineswegs eine „Republik ohne Republikaner“ war, sondern bis zum letzten Moment konsequente Verteidiger besaß, die eben nicht, wie das Urteil lange lautete, widerstandslos den Untergang der ersten Demokratie hinnahmen.

\title{
Viñas, Ángel: Quién quiso la guerra civil? Historia de una conspiración, 504 S., Editorial Crítica, Barcelona 2019.
}

\section{Reiner Tosstorff}

Online publiziert: 22. September 2020

(C) Der/die Autor(en) 2020

Ángel Viñas, seit 2011 emeritierter Lehrstuhlinhaber der Madrider Universität, hat eine überaus produktive Karriere im Wechsel zwischen der Zeitgeschichtsschreibung und den Wirtschaftswissenschaften absolviert. Sie ist, wie nicht nur das jetzt vorgelegte Buch zeigt, noch keineswegs beendet. Sein großes historisches Thema ist der Spanische Bürgerkrieg, dessen Ursprünge, der internationale Kontext und die Rolle des Diktators Francisco Franco. Und bei der Suche nach weltweiten Archivbeständen dürfte ihm auch sein alternierendes berufliches Standbein mit zeitweiligen Tätigkeiten in der spanischen Diplomatie, etwa als Wirtschaftsattaché in Bonn oder in der Europäischen Kommission, sehr nützlich gewesen sein.

Nach dem von der Diktatur Francos eifrig propagierten Geschichtsbild habe es sich bei dem Putsch um einen ,rein patriotischen“ Akt ohne internationale Verwicklungen gehandelt, um eine Reaktion auf einen maßgeblich von außen, nämlich von der Sowjetunion, gesteuerten Revolutionsversuch. Die Führungsposition General Francos habe gleichsam nahegelegen. Erst nach Putschbeginn sei angesichts der schwachen Ausgangsposition gar nichts anderes übrig geblieben, als die Unterstützung Deutschlands und Italiens zu suchen.

Bereits in seiner Doktorarbeit hat Viñas sich mit der Rolle der Nazi-Diktatur im Spanischen Bürgerkrieg beschäftigt und ist zu dem Schluss gekommen, dass die deutsche Intervention tatsächlich erst nach Ausbruch der Kämpfe, auf eine persönliche Entscheidung Adolf Hitlers gegenüber einem Hilfsbegehren der Putschisten hin,

R. Tosstorff $(\bowtie)$

Johannes Gutenberg-Universität Mainz, Mainz, Deutschland

E-Mail: Reiner.Tosstorff@uni-mainz.de 
durchgeführt wurde. Diese Hilfe, die zudem in enger Zusammenarbeit mit Italien erfolgte, machte Francos Sieg allerdings erst möglich. Zugleich nutzte Deutschland die im Gegenzug eingeräumten Zugeständnisse Francos mit zur Vorbereitung auf den Zweiten Weltkrieg. Das ist auch heute der durch weitere Studien bestätigte Forschungsstand.

Im vorliegenden Buch geht Viñas nun weit in die Vorgeschichte des Bürgerkriegs zurück. Im Fokus stehen dabei weniger das Militär und die verschiedenen hohen Offiziere, die im Frühjahr 1936 die einzelnen Schritte in Vorbereitung auf den Putsch in Angriff nahmen. Stattdessen geht er auf die Anfänge der Republik im April 1931 ein und blickt dabei auf die Monarchisten, die faktisch schon vom ersten Tag an zu deren Sturz entschlossen waren. Sie waren hohe Funktionsträger der Monarchie gewesen, insbesondere in ihrer Endphase ab 1923, einer Militärdiktatur unter General Primo de Rivera, aber weiterhin mit König Alfons XIII. als Staatsoberhaupt. $\mathrm{Zu}$ deren Stützung wurde eine Einheitspartei nach dem Vorbild des faschistischen Italiens geschaffen, auch wenn dem Regime eine vergleichbare gesellschaftliche Verankerung fehlte und es somit 1931 schnell zusammenbrach.

Aus diesen Kreisen rekrutierten sich die zum Putsch für die monarchische Restauration entschlossenen Kräfte unter Führung des ehemaligen Finanzministers José Calvo Sotelo. Dafür suchten sie Bündnispartner: in der auf einen anderen Prätendenten ausgerichteten monarchistischen Fraktion der Karlisten, in der katholischen Rechten der Sammelpartei CEDA (Confederación Española de Derechas Autónomas) und dann auch in der ab 1933 aktiven direkten faschistischen Organisation Falange. Vor allem aber knüpften sie Kontakte zu den Militärs, aus deren Reihen 1932 unter Führung von General José Sanjurjo ein erster Putschversuch gekommen war.

Viñas zeichnet nun in allen Einzelheiten die verschiedensten Schritte der sich entwickelnden Verschwörung nach. Insbesondere mit der Verschärfung des innenpolitischen Klimas nach den Neuwahlen Ende 1933, die einen scharfen Rechtsruck mit sich brachten, nahm das Komplott Fahrt auf. Der Kreis der Unterstützer wurde vor allem im Militär, aber auch unter den zivilen Kräften verbreitert. Schnell stellte sich heraus, dass man bei der Waffenbeschaffung ausländische Hilfe heranziehen musste. Dafür bot sich das faschistische Italien an, das so etwas wie ein politisches Vorbild in der Kombination von Monarchie und faschistischer Regierung wurde, und sich aus ideologischen wie aus geopolitischen Erwägungen - ein potenzieller Verbündeter gegen Frankreich und vor allem Großbritannien - interessiert zeigte. Schließlich kam es fast unmittelbar vor dem Putsch im Juli 1936 sogar zu einem regelrechten Abkommen mit Italien über die Lieferung von Flugzeugen.

Auf die Titelfrage seines Buchs nach denjenigen, die den Bürgerkrieg wollten, gelingt es Viñas, quellengesättigt zu antworten und damit die Geschichte der Verschwörung - so der Untertitel - nachzuzeichnen. Hinsichtlich der Fragestellung, warum denn der mit dem Resultat des Bürgerkriegs so eng verbundene Caudillo des neuen Regimes dabei lange Zeit allenfalls am Rande auftauchte, kann er dabei die Erkenntnisse neuerer Forschungen bestätigen, dieser habe bei der mehrjährigen Vorbereitung der Verschwörung keine Rolle gespielt und sei erst zum Schluss, wenige Monate vor dem Putsch - dann allerdings sehr tatkräftig - hinzugestoßen. So war Franco in der Planung des Aufstandes für keine herausragende Rolle in dem 
zukünftigen, zunächst allerdings nur vage skizzierten Regime vorgesehen. Es waren eine Reihe von Zufällen und der spezielle Verlauf der Kämpfe, die ihn nach einigen Monaten an die Spitze der Diktatur brachten. Was dem Autor dabei ebenfalls gelingt, ist der Nachweis der intensiven Bemühungen der doch so nationalpatriotisch auftretenden Verschwörer um ausländische Unterstützung. Damit praktizierten sie genau das, was sie zu ihrer eigenen Rechtfertigung ihrer Gegenseite, der Regierung der Republik, unterstellten.

Die vielen Einzelheiten in Viñas' Darstellung, für die er zahlreiche bisher unbekannte oder nicht ausreichend gewürdigte Dokumente aus einer ganzen Reihe von Archiven herangezogen hat, können hier nur in ihren wesentlichen Punkten aufgeführt werden. Darüber hinaus schreibt der Autor auch in einem manchmal etwas üppigen, sehr um das didaktische Wirken seiner Erkenntnisse bemühten Stil. Bei aller wissenschaftlichen Arbeitsweise wendet er sich vor allem an eine in Spanien aus offensichtlichen Gründen am Thema interessierte breite Leserschaft, die nicht unbedingt eine akademische Fachdiskussion führen will.

Wie auch schon die bisherigen Publikationen von Ángel Viñas ist das Buch in Spanien mit großem Echo aufgenommen worden. Das vorliegende Rezensionsexemplar weist die Angabe „sechster Druck“ - bereits ein halbes Jahr nach dem Ersterscheinen - auf. Das ist nicht verwunderlich. Die memory wars bilden heute in Spanien ein wichtiges Element der politischen Polarisierung. Dieses große Interesse ist angesichts der Art und Weise, wie das Regime im Elitenpakt zwischen Diktatur und Opposition 1977/78 durch offizielles Beschweigen beerdigt wurde, keine Überraschung. Noch immer gibt es auf der einen Seite mit dem Verblassen der Erinnerung an die Diktatur, verstärkt durch den Generationswechsel, ein überraschend breites Echo für neofranquistische Mythen, um den gegenwärtigen Krisenrealitäten eine Art ,heile Vergangenheit" gegenüberzustellen. Dagegen ist Viñas einer der wichtigen Zeithistoriker des Landes, denen es auf der anderen Seite immer wieder gelingt, die Geschichte durch das Graben in den Archiven mit neuen Funden richtigzustellen. Erfreulicherweise findet auch das bei einem erheblichen Teil der spanischen Gesellschaft seinen Widerhall.

Funding Open Access funding provided by Projekt DEAL.

Open Access Dieser Artikel wird unter der Creative Commons Namensnennung 4.0 International Lizenz veröffentlicht, welche die Nutzung, Vervielfältigung, Bearbeitung, Verbreitung und Wiedergabe in jeglichem Medium und Format erlaubt, sofern Sie den/die ursprünglichen Autor(en) und die Quelle ordnungsgemäß nennen, einen Link zur Creative Commons Lizenz beifügen und angeben, ob Änderungen vorgenommen wurden.

Die in diesem Artikel enthaltenen Bilder und sonstiges Drittmaterial unterliegen ebenfalls der genannten Creative Commons Lizenz, sofern sich aus der Abbildungslegende nichts anderes ergibt. Sofern das betreffende Material nicht unter der genannten Creative Commons Lizenz steht und die betreffende Handlung nicht nach gesetzlichen Vorschriften erlaubt ist, ist für die oben aufgeführten Weiterverwendungen des Materials die Einwilligung des jeweiligen Rechteinhabers einzuholen.

Weitere Details zur Lizenz entnehmen Sie bitte der Lizenzinformation auf http://creativecommons.org/ licenses/by/4.0/deed.de. 